\title{
Methods for assessment of vocal characteristics in dental treatment
}

\author{
Stoykov M., Milkov M., Peev S. \\ Faculty of Dental medicine, Medical University - Varna
}

\begin{abstract}
:
A phonetically correct articulation after teeth treatment and prosthetics is an important decision for a socially comfortable, meaningful, and high quality life. Dental treatment and prosthetics contribute to the preservation of normal speech ability, beyond just contributing to the preservation and re-establishing of the masticatory and aesthetic functions. While the last two roles of dental treatment and prosthetics are well understood, the phonetic recovering is still poorly comprehended and rather unvalued. The purpose of this article is to review the methods for assessing voice characteristics in dental treatment.
\end{abstract}

Key words: vocal characteristics, dental treatment, methods, assessment

\section{Introduction}

Proper knowledge of phonetics enables dentists to fabricate a prosthesis, which encounters the key objective of oral rehabilitation. (19) Voice quality (VQ) can be broadly defined as a combination of laryngeal and supralaryngeal characteristics in someone's voice, which leads to a long-term effect on perception and makes that voice recognizably different from the others. (16) Methodologically, assessment of VQ can be approached from an articulatory, acoustic or perceptual point of view. The use of phonography to distinguish vocal changes from normal voices has been documented in the literature $(14,18,24)$ to list changes in the voice due to fatigue and to assess changes in voice quality after voice therapy (13) or vocal singing training $(29,30)$. In particular, with regard to the standardization of reference values, there are population studies in Thailand (4), Germany (9) and Finland. (17)

Research also shows that there is a link between vocal tract dimensions and voice quality characteristics $(6,23)$; between voice disorders and oral muscle disorders $(8,25)$, as well as a relationship between the severity of temporomandibular disorders and vocal quality. (28)

Although literature demonstrates a link between the characteristics of the stomatognathic system and phonator function, no studies have been found to analyze vocal parameters, taking into account facial type and balanced occlusal condition importance. 
Aim: To review the methods for assessing voice characteristics in dental treatment.

\section{Material and methods}

The following research databases were used for the study: MEDLINE, Scopus, EMBASE and PubMed from January 2001 to August 2020, focusing on finding methods for assessing changes in voice characteristics in the field of prosthetic dental medicine.

\section{Results and discussion}

A phonetically correct articulation after teeth treatment and prosthesing plays an important role for a socially comfortable, meaningful, and high quality life. Dental treatment and prosthetics contribute to the preservation of the normal speech ability, beyond contributing just to the preservation and re-establishing of the masticatory and aesthetic functions. While the last two roles of dental treatment and prosthetics are well understood, the phonetic recovering is still poorly comprehended and rather unvalued.

Phonography is a measure used to record all the capabilities of the larynx in relation to the frequency and intensity of sound, as it covers the highest and lowest frequencies and intensities that the human larynx is capable of producing. (26)

Auditory-Perceptual Evaluation of Voice. The term perceptual evaluation is recommended by the Voice Committee of the International Association of Logopedics and Phoniatrics (IALP) and entails a comparison between the characteristics of the voice of the speaker and those that are considered normal or typical for the listener. (7) Since we are focused on the audible characteristics of the voice, we should point out that many authors expanded this term to auditory-perceptual evaluation. Clinicians and researchers believe that this form of perceptual evaluation is an essential component of voice assessment, diagnosis, and treatment. (5) The most commonly used perceptual evaluation systems have many similarities in terms of the voice features evaluated and definitions of those features. The GRBAS (Grade, Roughness, Breathiness, Asthenia and Strain) (12), CAPE-V (Consensus Auditory-Perceptual Evaluation of Voice) (1), Stockholm Voice Evaluation Approach (11) and the Perceptual Voice Profile (22), for instance, all incorporate the perceptual features.

Perceptual assessment. Speech-language patholo- gists are increasingly being encouraged to use the new Consensus Auditory-Perceptual Evaluation of Voice (CAPE-V) for clinical assessment of voice quality. It provides a standardized framework and procedures for perceptual evaluation of abnormal voice quality that include prescribed speech materials and visual analog scaling of a closed set of perceptual vocal attributes: overall severity of dysphonia, roughness, breathiness, straining, pitch and loudness. (1)

Acoustics assessment. Validity and reliability of acoustic measures currently used in the clinic to objectively assess voice quality (e.g., jitter, shimmer, and noise-to-harmonics ratio) are inherently limited by a reliance on the accurate determination of fundamental frequency (F0), and these measures have been further restricted to the analysis of sustained vowels. (36) One set of approaches is based on cepstral analysis (a spectrum-type method), which is inherently attractive since the cepstrum can be computed for any segment of speech and not just for steady vowel-like sounds. $(2,20,21)$

The second set of recently described acoustic voice measures is based on nonlinear dynamics or chaos analysis (35), which are much more robust with respect to analyzing atypical signals (e.g., aperiodic signals from pathological voices) than the measures currently used for clinical voice assessment. Aerodynamic assessment. Since the early 1980s, clinical assessment of aerodynamic voice parameters has typically involved extracting assessments of average subglottal air pressures and glottal air flow rates from non-invasive measures of intraoral air pressures and oral air flow rates during the controlled (constant pitch and loudness) repetition of simple syllable strings. It was subsequently shown that important additional information about glottal phonatory status (including the presence of pathology) could be obtained from estimates of the minimum air pressures required to initiate the softest possible voice production - the phonation threshold pressure. Further work using mathematical and physical laryngeal models has further demonstrated that phonation threshold pressure is sensitive to vocal fold thinning, viscous shear properties of the tissue, and vocal tract inertance. (3)

Yan and colleagues have used Nyquist plots, nonlinear processing of both the glottal area extracted from high-speed imaging and from the acoustic signal, to discriminate normal versus pathological voices. $(33,34)$ The quantification of glottal vari- 
ations at a high temporal resolution has also laid the ground work for more sophisticated computer models that can simulate asymmetric vocal fold tissue motion observed in excised larynx preparations (31) and live human subjects. $(27,32)$

Hamlet, Geoffrey and Bartlett (10) have analyzed the changes of voice characteristics due to dental prostheses by fitting subjects with experimental dental appliances that artificially lower and retract the alveolar-palatal contour. These authors analyzed acoustically and perceptually the subjects speaking without the prosthesis and with the prosthesis, in the first day and after a week of prosthesis wearing. They determined that the intelligibility of speech was significantly decreased by prosthesis wearing, especially before the subject had time to adapt to the new condition. Also, these authors found significant sibilant and vowel changes produced due to prosthesis wearing.

Laine (15) analyzed the associations between articulatory disorders and occlusal anomalies in young adults. The main findings are that several types of malocclusions, like mesial molar occlusion, mandibular overjet and lateral crossbite are associated with incorrect articulation of some medio-alveolar consonants, especially /s/. Laine argues that the analyzed occlusal anomalies affect speech primarily by changing the position of the tongue and hyoid bone.

While progresses have been made in the analysis of the speech quality lowering and in understanding some of the involved mechanisms, the domain is still in its infancy. The main needs are for relating acoustic analysis to the characteristics of the prostheses and to their design.

Correlation of the vocal signal changes with the characteristics of the prosthesis and the specific types of errors in the prosthetic act would be an essential achievement in the way of improving the outcome of the prosthetic act.

\section{Conclusion}

We defined several objectives and specific study methods and means for a new field that we called phonetic dentistry. Several plausible mechanisms that affect the phonetic quality of the prosthetic result have been derived based on articulatory mechanisms and on morphologic factors resulting from dentition deficiencies. The relationships between the articulatory facts and the acoustic features have been emphasized and discussed in order to derive acoustic methods and acoustic quantitative indices for assessing the dentition defects.

Some of the correlations between the acoustic features and the dentition deficiencies are already documented in the literature, but much remains to be done for establishing a corpus of knowledge and tools that could constitute the solid foundation of the emerging domain we call phonetic dentistry or gnathophonetics.

\section{References:}

1. American Speech-Language-Hearing Association: Consensus Auditory-Perceptual Evaluation of Voice (CAPE-V). Rockville Pike, American Speech-Language-Hearing Association, 2002

2. Awan SN, Roy N. Toward the development of an objective index of dysphonia severity: A four-factor acoustic model. Clinical Linguistics and Phonetics. 2006;20:35-49

3. Chan RW, Titze IR. Dependence of phonation threshold pressure on vocal tract acoustics and vocal fold tissue mechanics. Journal of the Acoustical Society of America. 2006;119:2351-2362

4. Chen SH. Voice range profile of Taiwanese normal young adults: a preliminary study. Zhonghua Yi Xue Za Zhi (Taipei). 1996;58(6):414-20

5. De Bodt MS, Wuyts FL, Van de Heyning PH, Croux C: Test-retest study of the GRBAS scale: influence of experience and professional background on perceptual ratings of voice quality. J Voice 1997;11:74-8

6. Dimitriev L, Kiselev A. Relationship between the formant structure of different types of supraglottic cavities. Folia Phoniat. 1979;32:238-41

7. Fex S: Perceptual evaluation. J Voice 1992;6: 155-158

8. Garcia RAS, Campiotto AR. Distúrbios vocais x distúrbios musculares orais: possíveis relaçöes. Pró-fono. 1995;7(2):33-9

9. Hacki T. Vocal capabilities of nonprofessional singers evaluated by measurement and superimposition of their speaking, shouting and singing voice range profiles. HNO. 1999;47:809-15

10. Hamlet SL, Geoffrey VC, Bartlett DM, Effect of a Dental Prosthesis on Speaker-Specific Characteristics of Voice, Dec. 1976d. J. Speech and Hearing Research Vol. 19, pp. 639-650

11. Hammarberg B, Gauffin J: Perceptual and acoustic correlates of quality differences in pathological voices as related to physiological aspects; in Fujimura O, Hirano M: Vocal Fold Physiology. Voice Quality Control. San Diego, Singular, 1995, pp 283-303

12. Hirano M: Clinical Examination of Voice. New York, Springer, 1981, pp 83-84

13. Holmberg EB, Ihre E, Sodersten M. Phonetograms as a tool in the voice clinic: changes across voice therapy for patients with vocal fatigue. Logoped Phoniatr Vocol. 2007;32(3):113-27

14. Ikeda Y, Masuda T, Manako H, Yamashita H, Yamamoto T, Komiyama S. Quantitative evaluation of the voice range profile in patients with voice disorder. Eur Arch Otorhinolaryngol. 1999;256:S51-5 
15. Laine T., Malocclusion traits and articulatory components of speech. European J. Orthodontics 1992 14(4):302-309

16. Laver J. The phonetic description of voice quality. Cambridge Studies in Linguistics London. 1980;31:1-86

17. Leino T, Laukkanen AM, Ilomäki I, Mäki E. Assessment of vocal capacity of finnish university students. Folia Phoniatr Logop. 2008;60(4):199-209

18. Ma E, Robertson J, Radford C, Vagne S, El-Halabi R, Yiu E. Reliability of speaking and maximum voice range measures in screening for dysphonia. J Voice. 2007;21(4):397-406

19. Moustafa AH.Clinical Complete Denture Prosthodontics, Post Insertion Denture Problems,Ist edition.King Saud University 2004;388

20. Murphy PJ. On first rahmonic amplitude in the analysis of synthesized aperiodic voice signals. Journal of the Acoustical Society of America. 2006;120:2896-2907

21. Murphy PJ, Akande OO. Noise estimation in voice signals using short-term cepstral analysis. Journal of the Acoustical Society of America. 2007;121:1679-1690

22. Oates J, Russell A: Learning voice analysis using an interactive multi-media package: development and preliminary evaluation. J Voice 1998; 12:500-512

23. Oliveira VL, Pinho SMR. A qualidade da voz e o trato vocal nos indivíduos de face curta e face longa. In: PINHO, S.M.R. Tópicos em voz. Rio de Janeiro: Guanabara Koogan, 2001

24. Pribuisiene R, Uloza V, Saferis V. Multidimensional voice analysis of reflux laryngitis patients. Eur Arch Otorhinolaryngol. 2005;262(1):3540

25. Ringel RL, Chodzko-Zajko WJ. Vocal indices of biological age. J. Voice. 1987;1:31-7

26. Schutte HK, Seidner W. Recommendation by the Union of European Phoniatricians (UEP), Folia-Phoniatr-(Basel). 1983;35(6):286-8

27. Schwarz R, Hoppe U, Schuster M, et al. Classification of unilateral vocal fold paralysis by endoscopic digital high-speed recordings and inversion of a biomechanical model. IEEE Transactions on Biomedical Engineering. 2006;53:1099-1108

28. Silva AMT, Morisso MF, Cielo CA. Relationship between the severity of temporomandibular disorder and voice. Pró-Fono. 2007;19(3):27988

29. Siupsinskiene N. Quantitative analysis of professionally trained versus untrained voices. Medicina. 2003;39(1): 36-46

30. Sulter AM, Schutte HK, Miller DG. Differences in phonetogram features between male and female subjects with and without vocal training. J Voice. 1995;9(4):363-77

31. Tao C, Zhang Y, Jiang JJ. Extracting physiologically relevant parameters of vocal folds from high-speed video image series. IEEE Transactions on Biomedical Engineering. 2007;54:794-801

32. Wurzbacher T, Schwarz R, Döllinger M, et al. Model-based classification of nonstationary vocal fold vibrations. Journal of the Acoustical Society of America. 2006;120:1012-1027

33. Yan Y, Chen X, Bless D. Automatic tracing of vocal-fold motion from high-speed digital images. IEEE Transactions on Biomedical Engineering. 2006;53:1394-1400

34. Yan YL, Damrose E, Bless D. Functional analysis of voice using simultaneous high-speed imaging and acoustic recordings. Journal of Voice. 2007;21:604-616

35. Zhang Y, Jiang JJ. Acoustic analyses of sustained and running voices from patients with laryngeal pathologies. Journal of Voice. 2008;22:1-9

36. Zeitels SM, Blitzer A, Hillman RE, et al. Foresight in laryngology and laryngeal surgery: A 2020 vision. Annals of Otology, Rhinology, and Laryngology Supplement. 2007;198:2-16 